\title{
Status Of Pulsed Inductive Thruster Research
}

\author{
Ivana Hrbud ${ }^{1}$, Michael LaPointe $^{2}$, Robert Vondra ${ }^{3}$, C. Lee Dailey ${ }^{4}$, Ralph Lovberg ${ }^{5}$ \\ ${ }^{\prime}$ NASA Marshall Space Flight Center, TD40, Huntsville, AL 35812 \\ ${ }^{2}$ Ohio Aerospace Institute/NASA Glenn Research Center, \\ ${ }^{3}$ TRW, Inc., Redondo Beach, CA \\ ${ }^{4} R L D$ Associates, Inc. \\ ${ }^{5}$ RLD Associates, Inc. \\ (256) 544-2465, Ivana.Hrbud@msfc.nasa.gov
}

\begin{abstract}
The TRW Pulsed Inductive Thruster (PIT) is an electromagnetic propulsion system that can provide high thrust efficiency over a wide range of specific impulse values. In its basic form, the PIT consists of a flat spiral coil covered by a thin dielectric plate. A pulsed gas injection nozzle distributes a thin layer of gas propellant across the plate surface at the same time that a pulsed high current discharge is sent through the coil. The rising current creates a time varying magnetic field, which in turn induces a strong azimuthal electric field above the coil. The electric field ionizes the gas propellant and generates an azimuthal current flow in the resulting plasma. The current in the plasma and the current in the coil flow in opposite directions, providing a mutual repulsion that rapidly blows the ionized propellant away from the plate to provide thrust. The thrust and specific impulse can be tailored by adjusting the discharge power, pulse repetition rate, and propellant mass flow, and there is minimal if any erosion due to the electrodeless nature of the discharge. Prior single-shot experiments performed with a 1-meter diameter version of the PIT at TRW demonstrated specific impulse values between 2,000 seconds and 8,000 seconds, with thruster efficiencies exceeding $55 \%$ for ammonia and hydrazine propellants. This paper outlines current and planned activities to transition the single shot device into a multiple repetition rate thruster capable of supporting future DOD and NASA strategic enterprise missions.
\end{abstract}

\section{INTRODUCTION}

Rapid access to any point in the solar system and beyond requires advanced propulsion concepts that can provide extremely high specific impulse, high specific power, and high thrust-to-power ratios. NASA's vision for the $21^{\text {st }}$ century and beyond is challenging scientific and engineering communities to develop propulsion technologies which will enable ambitious exploration of the solar system and its interstellar neighborhood, commercialization of space, and eventual human colonization beyond Earth (Schmidt, 1998). Two technologies that synergistically offer tremendous potential for the immediate future are advanced electric propulsion and nuclear energy. Space nuclear systems have recently received a renewed interest due to their versatility, high-power density, and ability to support power-intensive missions (Borowski et al., 1998). The duality of providing both propulsion and power, linked with the enormous energy available per unit mass of fission fuel, has significant benefits to future programs involving nuclear thermal rockets, efficient conversion systems, space-based nuclear reactors, and nuclear electric propulsion (Allen et al., 1995).

To date, only a few electric propulsion concepts can support the high power requirements for future missions. These are the magnetoplasmadynamic (MPD) thruster (Polk, 1999, LaPointe and Sankovic, 2000), the Variable Specific Impulse Magnetoplasma Rocket (VASIMR) (Chang-Diaz, 1995), advanced ion and Hall-ion thrusters (Sankovic et $a l$.), and the pulsed inductive thruster (PIT) (Dailey and Lovberg, 1993). Because of it's variable repetition rate and electrodeless design, the PIT appears to be an ideal candidate for high power electric propulsion missions powered by solar or in-space nuclear power.

Research in the field of pulsed electromagnetic acceleration has been conducted since the early 1960 s, resulting in a number of different pulsed devices capable of supporting various propulsion requirements. These devices use either a pair of suitable electrodes or electrodeless techniques to couple energy into a plasma. In either case, the strong interaction of plasma currents and induced magnetic fields governs the acceleration mechanisms of the ionized propellant. Two of the most prominent examples of pulsed thrusters are the pulsed plasma thruster (PPT), a very low power device supporting near-Earth missions which require small, precise impulse bits (Benson et al., 1999), 
and the pulsed inductive thruster (PIT), a multi-megawatt device potentially capable of supporting human/robotic missions to Mars and the outer solar system.

The pulsed inductive thruster was first developed at TRW during the 1960s, with support from the Department of Defense and a significant investment of internal TRW research and development funds (Dailey and Lovberg, 1987, 1993). Preliminary thruster designs with coil diameters of $20 \mathrm{~cm}$ and $30 \mathrm{~cm}$ were first investigated for low power applications, but because of parasitic circuit and other losses they achieved efficiencies of less than $20 \%$ (Dailey et al., 1971, 1973). During the 1970s and 1980s, TRW continued to investigate and improve thruster operation, establishing scaling laws that could be used to characterize thruster performance with respect to coil size and power input. During the early 1990s, TRW received additional funding from the NASA Glenn Research Center to conduct a series of high power discharge tests. The performance of the now standard 1-m diameter PIT under single-shot (non-repetitive) operation demonstrated significantly improved thruster performance, reaching efficiencies of around $50 \%$ and specific impulse values of over $7000 \mathrm{~s}$. Unfortunately, agency funding for high power electric propulsion declined in the years that followed, and the PIT was mothballed at TRW for several years. With the recent advent of the NASA Human Exploration and Development of Space (HEDS) and Space Science enterprise missions to Mars and the outer planets, interest has once again turned to the pulsed inductive thruster as an efficient high power propulsion alternative. As a result, a collaborative effort was initiated in 1999 between TRW, Inc., the NASA Glenn Research Center, and the NASA Marshall Space Flight Center to develop and test a repetitively pulsed version of the PIT. This paper provides a status review of the collaborative effort, with particular emphasis on the preliminary design results for a solid state switching circuit and program plans for the development and testing of a high power, multiple repetition-rate version of the thruster.

\section{PULSED INDUCTIVE THRUSTER}

\section{Operating Principle}

The Pulsed Inductive Thruster (PIT) is characterized by $\mu$-second, MW-power pulsed operation that can provide high thrust efficiency over a wide range of specific impulse values. The main components of the thruster include a flat spiral inductance coil covered by a thin insulator, a propellant injection nozzle that extends above the coil, and a pulsed power supply consisting of a capacitor array, switch assembly and pulse forming network. The nozzle injects a puff of propellant gas that covers the coil surface, while the pulse forming network simultaneously triggers the discharge of the high-energy capacitor bank. The transient high current pulse passes through the coil, generating a rapidly changing magnetic field that induces a strong azimuthal electric field in the region above the coil. The propellant is ionized by the electric field, and an azimuthal current is generated in the plasma to oppose the rising current in the coil. The interaction between the two counterpropagating current loops forces the plasma axially away from the coil to produce thrust. The thrust and specific impulse can be tailored by adjusting the discharge power, pulse repetition rate, and propellant mass flow. There is minimal if any component erosion due to the electrodeless nature of the discharge. Figure 1 illustrates the PIT thruster as it was designed and tested at TRW.

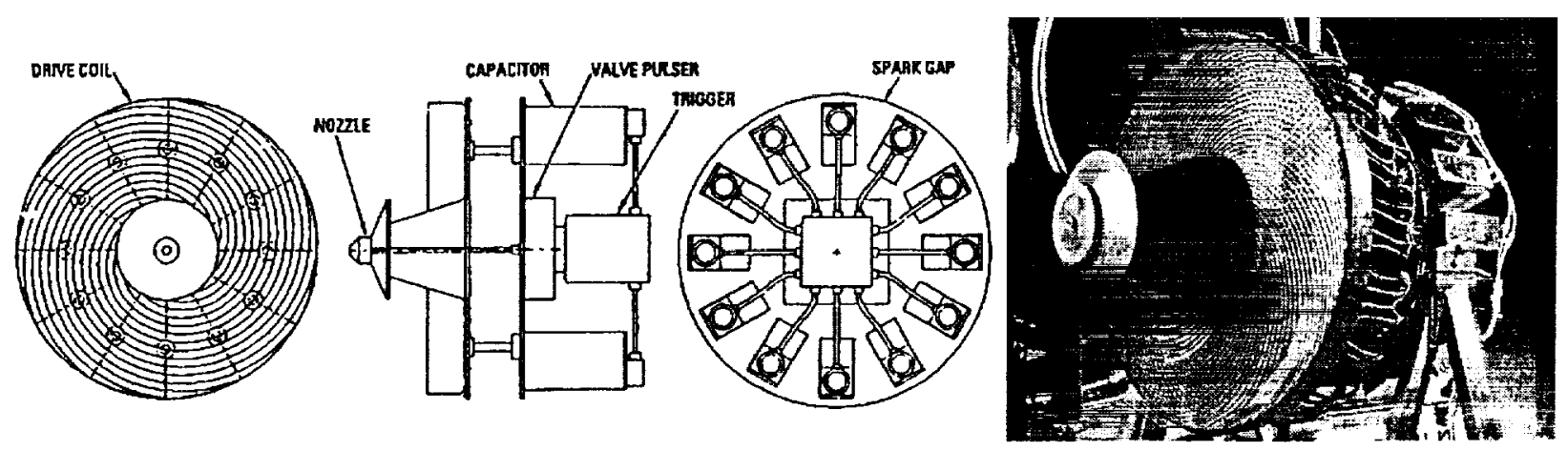

Figure 1. Pulsed Inductive Thruster developed at TRW. 


\section{Characteristic Performance Parameters}

The push for large scale PIT thruster research was initiated due to 1) the necessity for higher power, efficiency and thrust demanded for emerging power intensive space missions, and 2) the results of numerical analysis showing higher efficiencies with increasing coil size. In the most recent study sponsored by NASA Glenn Research Center, TRW evaluated the performance of a I-meter diameter PIT thruster (Dailey and Lovberg, 1993). The coil of the thruster was supplied with energy stored in a Marx bank network of eighteen 20-kV capacitors. The bank configuration consisted of nine circuits in parallel, with two capacitors per circuit. The Marx bank concept allows charging a capacitor array (consisting of n-number of units) in parallel and discharging the array in series connection. Charging can thus be accomplished at a much lower voltage, which is typically safer and requires simpler operating circuitry. Voltage multiplication due to the series connection of capacitor units furnishes the high operating voltage required for thruster discharge, without the need for voltage converters or step-up transformers. With each capacitor having a capacitance of $2 \mu \mathrm{F}$, the Marx bank configuration yielded an effective capacitance of 9 $\mu \mathrm{F}$. The total inductance of the circuit was $740 \mathrm{nH}$. The life rating was $10^{4}$ pulses, and the reversal capability was assessed at $40 \%$ when operated at the maximum design voltage. Charging the capacitors to less than $16 \mathrm{kV}$ extended their life rating to approximately $10^{5}$ pulses, but limited the total discharge energy of the bank to about 4.6 kJ. Performance characteristics were evaluated with a variety of propellants ranging from ammonia, hydrazine, and argon, to carbon dioxide. Table 1 summarizes the typical performance parameters obtained for various types of propellant, averaged over the reported charging voltages and injected propellant masses.

Table 1. PIT Performance for Various Propellants

\begin{tabular}{lcc}
\hline Propellant & Specific Impulse $[$ s] & Efficiency \\
\hline Ammonia & $3,000-8,000$ & $50 \%$ \\
Hydrazine & $2,500-5,000$ & $40 \%$ \\
Argon & $1,000-2,000$ & $20-30 \%$ \\
Carbon Dioxide & $1,000-2,000$ & $20-30 \%$ \\
\hline
\end{tabular}

To illustrate the potential capability of this thruster, Figure 2 shows measured thruster efficiency as a function of specific impulse and propellant mass per pulse. The efficiency remains at about $50 \pm 5 \%$ over a wide range of specific impulse values $(2,000-7,500 \mathrm{~s})$ and propellant mass increments $(0.8-7.5 \mathrm{mg})$. Figure 3 presents the measured thruster impulse as a function of the injected mass per pulse for a fixed capacitor charging voltage. As shown by Figures 2 and 3, varying the amount of injected propellant mass provides significant throttle ability, with very little deterioration of efficiency, for a fixed capacitor voltage and propellant type.

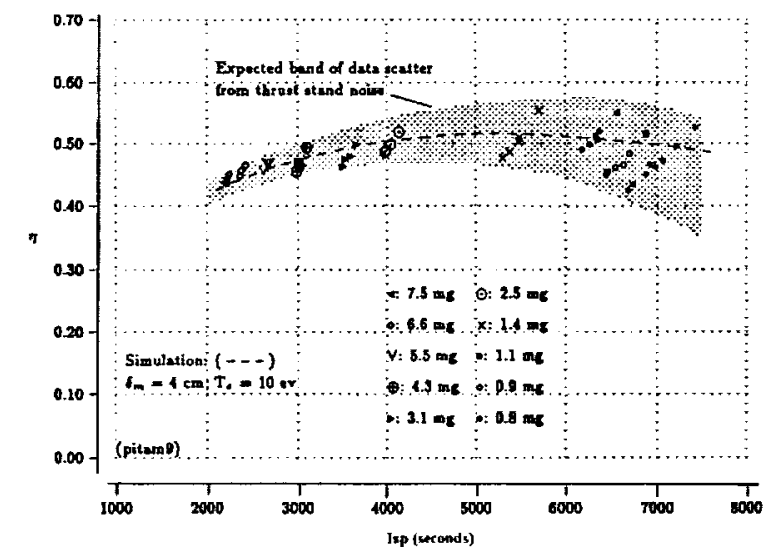

Figure 2. Efficiency as a Function of Specific Impulse for Ammonia at $15 \mathrm{kV}$.

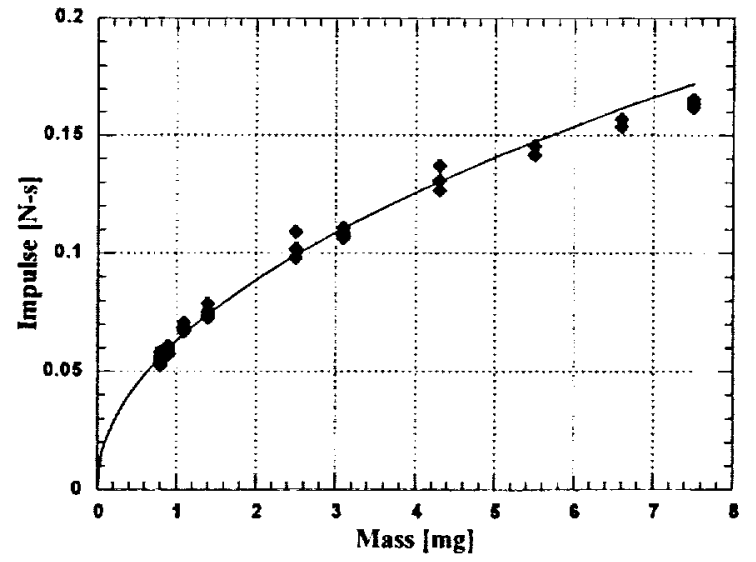

Figure 3. Impulse as a Function of Mass for Ammonia at $15 \mathrm{kV}$. 
The specific impulse and efficiency are independent of the thruster discharge frequency, but the repetition rate does determine the average thrust generated during repetitively pulsed operation. For the next generation PIT, the projected repetition rate is 10 to 100 pulses per second. Assuming an average impulse per discharge of around 0.1 $\mathrm{N}-\mathrm{s}$, the average thrust provided by the PIT operating at $10-\mathrm{Hz}$ is approximately $1-\mathrm{N}$, and $10-\mathrm{N}$ at an operating frequency of $100-\mathrm{Hz}$. The repetition rate is primarily determined by the amount of in-space power available to recharge the capacitor bank. The typical discharge energy per pulse is on the order of a few kilojoules per pulse, so a repetition rate of $10-\mathrm{Hz}$ would require a few tens of kilowatts of in-space power. At this power level, which can be achieved with standard solar arrays, the thruster can provide a few newtons of average thrust at a user-defined specific impulse of between $2000 \mathrm{~s}$ and $7000 \mathrm{~s}$, with a thruster efficiency of around $50 \%$. Higher repetition rates will in turn require higher in-space power capabilities, leading to the use of space nuclear reactors for several of the piloted and robotic deep space missions envisioned by NASA. The remarkable ability to provide variable specific impulse and thrust while maintaining a high overall thruster efficiency makes the PIT a unique device that is well suited for applications ranging from lunar and Mars cargo transport to future missions of planetary and deep space exploration.

\section{Disadvantages of Spark Gap Switching}

The PIT devices used in previous research efforts operated with high voltage spark gap switches to deliver current from the capacitor bank to the discharge coil. The discharge in a spark gap depends on a multitude of parameters such as electrode material, shape, gap distance, configuration, gas environment, and power characteristics. While suitable for single-shot ground tests, they have limited life and cannot be used in pulsed modes operating at high repetition rates. To support an interplanetary mission, the estimated lifetime requirement is about $10^{10}$ pulses (Dailey and Lovberg, 1993). Spark gap switches cannot meet this life requirement due to the erosion of the switch electrode material; at a nominal erosion rate of $10 \mu \mathrm{g} / \mathrm{C}$, a spark gap switch would erode more than one kilogram of tungsten over the life of the mission. This erosion would significantly alter the electrode gap separation, which is a critical parameter for switch operation and performance. In addition, radiative destruction of the insulating materials within the gap will occur, ultimately leading to the deterioration of spark gap performance. In most cases, especially for very high voltage applications, these switches require a high vacuum to ensure the proper hold-off voltage followed by precise gas injection at a given gap distance to carry the main discharge. Typically, a trigger electrode is introduced to provide enough preionization charge carriers to enable the main discharge, but a major difficulty in employing a parallel array of spark gaps is the stringent requirement to fire all switches simultaneously. The slightest time difference could lead to the firing of only one spark gap and removing voltage from the second spark gap before its trigger arrives. In this case the second gap would never fire at all, providing only partial discharge of the bank and poor thruster performance. For these reasons, solid-state switches capable of supporting high voltage a rapid current rise times are being considered for the next generation of Pulsed Inductive Thruster hardware.

\section{Next Generation PIT}

TRW, Inc., the NASA Glenn Research Center (GRC), and the NASA Marshall Space Flight Center (MSFC) have formed a collaborative research project to advance the state of the art pulsed inductive thruster. The primary technical objectives of this research and development program are to address key thruster design and performance issues, such as the development of a high rep-rate propellant-delivery system, improvements to the pulsed power network design, and physics-based modeling of high power thruster operation. The ultimate goal of this combined research effort is to design, build and ground-test a repetitively pulsed thruster that will serve as a transitional device for future MW-class, flight-qualified engines.

\section{Thruster Hardware.}

Following decades of research, the available PIT hardware (PIT-MkV) is unsuitable for further testing and must be replaced with new components better suited for repetitive operation. Under a recent 6-month NASA contract, TRW identified and designed several of the key components required for the next generation of repetitively pulsed inductive thrusters (Lovberg and Dailey, 2000). The proprietary contractor report will be used as a guideline for fabricating and testing the PIT-MkVI thruster, a MW-class breadboard device designed to operate in short bursts at 
high repetition rate. The new thruster hardware, which includes a $1-m$ diameter inductance coil, repetitively pulsed propellant valve, thrust balance, and support structures will be fabricated and tested at the TRW facility in Redondo Beach, CA. Although the basic appearance of the thruster will remain similar to the schematic shown in Figure 1, design modifications will be made to the to the $\mathrm{MkVI}$ model to support repetitively pulsed operation. The primary change in moving from the single shot PIT-MkV to repetitive burst mode operation with the PIT-MkVI is to replace the spark gap switches with high voltage, solid-state switches capable of sustaining high thruster repetition rates. The evaluation and incorporation of these solid-state switches into the PIT-MkVI design is the principle near-term focus of the collaborative effort, as discussed in the following section.

\section{Solid-State Switches.}

Figure 4 displays a typical current waveform measured for an individual PIT circuit element. The peak current is approximately $15 \mathrm{kA}$, and initial rise time $(\mathrm{dl} / \mathrm{dt})$ is on the order of $30 \mathrm{kA} / \mu \mathrm{s}$. The near-term objective of the PITMkVI solid-state switch effort is to evaluate commercially available switch performance with regard to these two key parameters. To test candidate high-power solid-state switch technology, two independent LRC circuits were designed, each addressing one key parameter. The simplified LRC circuits do not attempt to reproduce the full current waveform shown in Figure 4, but rather are used to evaluate peak current and rise time capabilities. Both circuits consist of LRC components connected in series. The capacitance is $2 \mu \mathrm{F}$ and the charging voltage is between 15 and $20 \mathrm{kV}$, in accord with the energy storage requirements for the original Marx bank configuration. The boundary conditions dictate the resistance and the inductance values for the two circuits. For peak current measurements, a period of about $10-15 \mu \mathrm{s}$ and a current reversal of $50 \%$ for the second half cycle of oscillation are required. To test $\mathrm{dl} / \mathrm{dt}$ capability, the inductance is determined according to $\mathrm{L}=\mathrm{V}_{0} /(\mathrm{dl} / \mathrm{dt})$, where $\mathrm{V}_{0}$ is the charge voltage of the capacitor, and resistance is calculated based on critical damping.

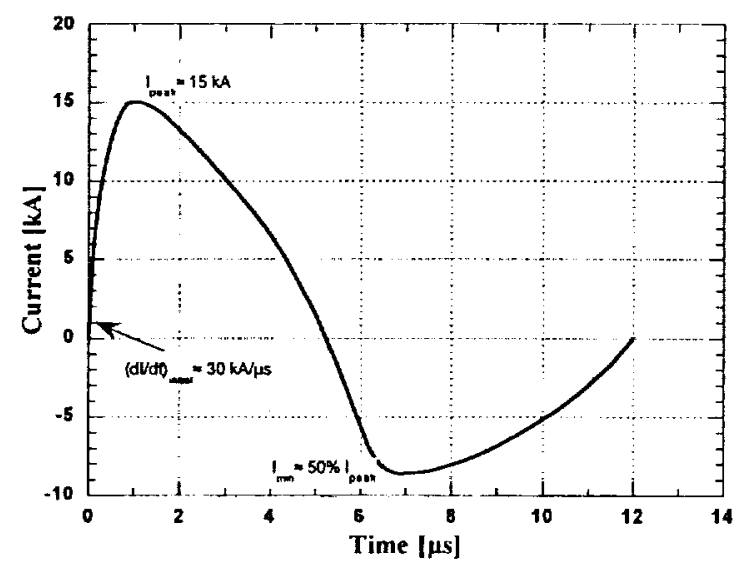

Figure 4. Current Waveform of one Circuit in Thruster Experiment.

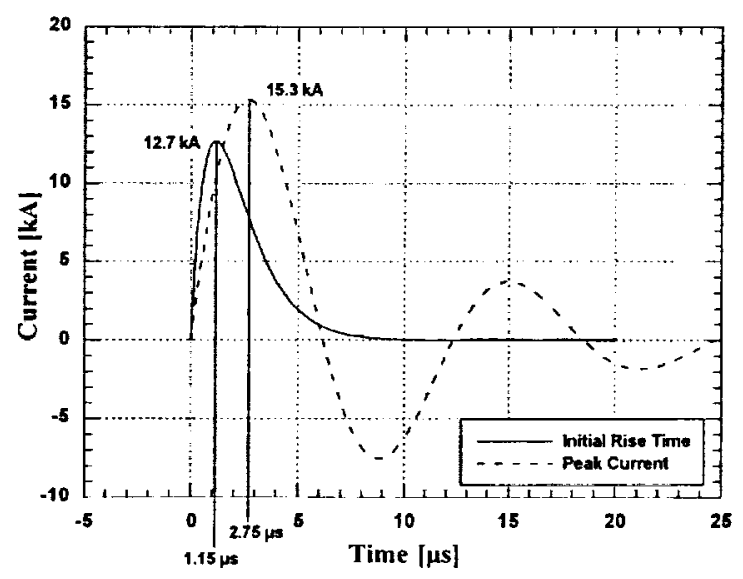

Figure 5. Analytical Results for Peak Current and Initial Rise time Testing.

Figure 5 shows analytical results assuming a charging voltage of $20 \mathrm{kV}$ for the two key parameters according to the discussed requirements. The component values for each LRC circuit are summarized in Table 2 for the expected voltage range.

Table 2. Analytical Results

\begin{tabular}{lcccc}
\hline & Voltage $[\mathbf{V}]$ & Capacitance $[\boldsymbol{\mu} \mathbf{F}]$ & Inductance $[\boldsymbol{\mu H}]$ & Resistance $[\boldsymbol{\Omega}]$ \\
\hline Peak Current Analysis & 20,000 & 2 & 1.836 & 0.423 \\
& 16,000 & 2 & 1.27 & 0.35 \\
Initial Rise Time Analysis & 20,000 & 2 & 0.667 & 1.158 \\
& 16,000 & 2 & 0.533 & \\
\hline
\end{tabular}


An industry survey is currently underway as a prelude to the acquisition and testing of suitable solid-state switches that can meet these requirements. Switch evaluations are expected to begin in FYOI, with final component selection and in-depth performance testing to occur in FY02.

\section{Concluding Remarks}

The pulsed inductive thruster is an efficient, versatile space transportation system that can enhance or enable a variety of NASA missions. As in-space power capabilities evolve to meet even more demanding mission requirements, the same fundamental thruster design can be used to support lunar, planetary and deep space exploration activities. Unlike current electric propulsion devices, the PIT is an electrodeless system that can operate efficiently over a wide range of specific impulse. In addition, its electrodeless nature mitigates material and component erosion, and the development of solid-state switches in concert with commercially available long-life capacitors ensures thruster longevity. The average thrust provided by the PIT is determined by the pulse repetition rate, which in turn depends on the available power required to charge the capacitors. Current solar arrays are sufficient to operate the PIT at a rep-rate of $10-\mathrm{HZ}$, providing an average thrust of around 1-N to support near-Earth and lunar cargo missions. As in-space power capabilities grow to encompass nuclear power, the highly efficient, variable specific impulse PIT becomes an attractive option for planetary and deep space applications. The combination of long life, high thrust, and variable specific impulse is unique among electric propulsion systems, making the PIT well suited for the challenging missions envisioned by NASA. To further develop this unique capability, TRW and NASA have teamed to develop the next generation of repetitively pulsed inductive thrusters, designated PIT-MkVI. Through a combined multi-year effort involving numerical simulation, component design, and breadboard thruster performance testing, it is anticipated that this next generation thruster will demonstrate the viability of efficient, high rep-rate operation in a simulated space environment, leading to the design and future deployment of flight qualified pulsed inductive thrusters.

\section{ACKNOWLEDGEMENTS}

This research is funded through the NASA Advanced Space Transportation Program, whose support is gratefully acknowledged.

\section{REFERENCES}

Allen, D. M., Bennett, G. L., El-Genk, M. S., Newhouse, A. R., and Rose, M. F., "Maintenance of the U.S. Space Nuclear Power Program: An AIAA Position Paper", $46^{\text {th }}$ International Astronautical Congress, IAF-95-R.1.05, Oslo, Norway, October 1995.

Benson, S. W., Arrington, L. A., Hoskins, W. A., and Meckel, N. J., "Development of a PPT for the EO-1 Spacecraft", 35 ${ }^{\text {th }}$ Joint Propulsion Conference, AIAA 99-2276, Los Angeles, CA, June 1999.

Borowski, S. K., Dudzinski, L. A., and McGuire, M. L., "Vehicle and Mission Design Options for the Human Exploration of Mars/Phobos Using Bimodal NTR and LANTR Propulsion", NASA/TM-1998-208834, December 1998.

Chang-Diaz, F. R., Braden, E., Johnson, I., Hsu, M. M., and Yang, T. F., "Rapid Mars Transits with ExhaustModulated Plasma Propulsion", NASA Technical Paper 3539, March 1995.

Dailey, C. L., "Pulsed Electromagnetic Thruster", AFRPL-TR-71-107, December 1971.

Dailey, C. L., Davis, H. A., and Hayworth, B. R., "Pulsed Plasma Propulsion Technology", AFRPL-TR-73-81, July 1973.

Dailey, C. L. and Lovberg, R. H., "Pulsed Inductive Thruster", Final Technical Report for Air Force Rocket Propulsion Laboratory, Contract No. F04611-82-C-0058, January 1987.

Dailey, C. L. and Lovberg, R. H., "The PIT MkV Pulsed Inductive Thruster", NASA CR 191155, July 1993.

LaPointe, M. R. and Sankovic, J. M., "High Power Electromagnetic Propulsion at the NASA Glenn Research Center", Proceedings of the Space Technologies and International Forum (STAIF) 2000 Conference, American Institute of Physics Conference Proceedings 504, M. El-Genk (ed), January 2000, pp. 1538-1543.

Lovberg, R. H. and Dailey, C. L., "Design of PIT MkVI", NASA/CR-2000-210573, November 2000.

Polk, J., Tikhonov, V., and Semenikhin, S., "200-kW Lithium-Fed Thruster Development and Preliminary Test", $35^{\text {th }}$ Joint Propulsion Conference, AIAA 99-2285, Los Angeles, CA, June 1999. 
Sankovic, J., Oleson, S. and LaPointe, M., "NASA GRC Activities in High Power Electric Propulsion", Proceedings of the NASA/JPL $10^{\text {th }}$ Advanced Space Propulsion Workshop, April 1999, pp. 665-681.

Schmidt, G. R., "Propulsion Research and Technology at NASA Marshall Space Flight Center", $34^{\text {th }}$ AIAA Joint Propulsion Conference, AIAA 98-3230, Cleveland, OH, July 1998. 